
\title{
R Reserach S Suare \\ DDoS Attack Detection Method under Deep Learning in the Context of SDN-OpenFlow
}

Gang Ke ( $\sim$ kegang95@126.com )

Dongguan Polytechnic

\section{Ruey-Shun Chen}

Dongguan Polytechnic

\section{Y.C. Chen}

University of California Davis

Naixue Xiong

Tianjin University

Yuan Tian

Nanjing Institute of Technology

Najla Al-Nabhan

King Saud University

\section{Research}

Keywords: DDoS attack detection, SDN-OpenFlow, Deep learning, Network security

Posted Date: April 24th, 2020

DOI: https://doi.org/10.21203/rs.3.rs-20700/v1

License: (c) (1) This work is licensed under a Creative Commons Attribution 4.0 International License.

Read Full License 


\section{Abstract}

The authors have removed this preprint from Research Square. 